\title{
S-adenosyl-L-methionine, trehalose and oleanolic acid in few plants
}

\author{
Dipankar Malakar, Paramita Chaudhuri, Trina Dutta, Anil K. Ghosh*
}

Biotechnology Department, Indian Institute of Chemical Biology, Kolkata, India; ${ }^{*}$ Corresponding Author: aghosh@iicb.res.in

Received 22 February 2010; revised 30 March 2010; accepted 2 April 2010.

\begin{abstract}
S-Adenosyl-L-Methionine (AdoMet), S-AdenosylL-Homocysteine (AdoHcy), adenosine, trehalose and oleanolic acid were measured in six medicinal herbs and three spices. The findings showed that AdoMet content was forty six fold higher in the leaves of Catharanthus roseus as compared with average AdoMet content of rest of the plants. In comparison to other plants, Withania somnifera had very high AdoHcy: AdoMet and adenosine: AdoMet ratios indicating it may have contained high AdoMet. Trehalose was found to be twenty fold and nine fold higher in bulb of Allium cepa and root of Withania somnifera respectively with respect to average trehalose content of rest of the plants. Ocimum sanctum appeared to be a rich source of oleanolic acid. It appeared from our study that $\mathrm{Ca}$ tharanthus roseus, Allium cepa and Ocimum sanctum could be utilized as natural sources of AdoMet, trehalose and oleanolic acid respectively.
\end{abstract}

Keywords: AdoMet; AdoHcy; Adenosine; Trehalose; Oleanolic Acid

\section{INTRODUCTION}

S-Adenosyl-L-Methionine (AdoMet) is the only natural sulfonium compound present in different living organisms. It is an important metabolic intermediate participating in different biochemical events and acts as a universal methyl group donor in transmethylation reactions, transsulfuration reactions and polyamine synthesis [1] The molecule has therapeutic importance in the treatment of alcoholic liver disease [2], cirrhosis of liver [3], depressive syndrome [4], Alzheimer disease [5], Osteoarthritis [6] etc. and may be classified as an "antiaging compound".
Trehalose ( $\alpha$-D-glucopyranosyl- $\alpha$-D-glucopyranoside) is a non reducing disaccharide having high glass transition temperature $\left(\mathrm{Tg}=80^{\circ} \mathrm{C}\right)$ is well known for effective stabilization of macromolecules like proteins [7], small molecules like AdoMet [8], etc. It also acts as a cryopreservative of cellular membranes [9].

Oleanolic acid is a triterpenoid compound that exists widely in food, medicinal herbs and other plants. It is effective in protecting against chemically induced liver injury in laboratory animals where the mechanism of hepatoprotection may involve the inhibition of toxicant activation and the enhancement of the body defense systems [10]. Oleanolic acid has also been long recognized to have antiinflammatory and antihyperlipidemic properties in laboratory animals [11]. But more research is warranted to develop a therapy for patients. Recently, oleanolic acid has been noted for its anti-tumor activity [12].

Extracts from the leaf of Ocimum sanctum [13], stem of Tinospora cordifolia [14], rhizosphere of Picrorrhiza kurroa [15], root of Withania somnifera[16], whole body (except root) of Eclipta erecta [17], bulbs of Allium sativum [18] and Zingiber officinale [19] are already proven for their hepatoprotective activities beside other therapeutic potentialities.

In the present communication, we have made comparative studies on AdoMet, trehalose and oleanolic acid content among the above mentioned medicinally important plants. For our experiments we have taken root of $W$. somnifera, leaf of $O$. sanctum, stem of $T$. cordifolia, rhizosphere of $P$. kurroa, whole body (except root) of $E$. erecta and bulbs of $A$. sativum and $Z$. officinale. The bulb of Allium cepa, which is well known for its food preservation activities and leaf of Catharanthus roseus [20] which have anti-tumor, anti-oxidative activities were also chosen for analysis.

\section{MATERIALS AND METHODS}

The experiments were performed in triplicate by col- 
lecting different parts of young herbs and spices from a particular area of eastern India in winter season so that within different experimental sets geological, environmental and chronological variations could be avoided. The intracellular matter from the specific parts of the different herbs and spices were simultaneously extracted and deproteinized [21] prior to the estimation of trehalose, AdoMet and its irreversibly transformed metabolic products AdoHcy and adenosine. Briefly, each of the herbs and spices were cut into small pieces and washed thoroughly with tap water.

\section{EXTRACTION AND DEPROTEINIZATION}

The washed pieces were then air-dried and $0.25 \mathrm{~g}$ (dry weight) of each of the plant parts was homogenized with $0.5 \mathrm{ml}$ of cold $0.5 \mathrm{~N}$ perchloric acid for deproteinization purpose. The mixture was centrifuged at $5,500 \times g$ for 10 min and the pellet was extracted further with $0.5 \mathrm{ml}$ of cold $0.2 \mathrm{~N}$ perchloric acid and again centrifuged. The process was repeated and the supernatants were pooled. The $\mathrm{pH}$ value of the pool was adjusted to 4.5 by careful addition of $3 \mathrm{~N} \mathrm{KOH}$. This caused precipitation of potassium per chlorate and the precipitate was removed by low speed centrifugation for 10 minutes at $4^{\circ} \mathrm{C}$. Careful reduction of volume under reduced pressure at relatively low temperature resulted in further precipitation of potassium per-chlorate and this was again removed by low speed centrifugation. The supernatants were dried and reconstituted with $1 \mathrm{ml}$ of $0.01 \mathrm{~N} \mathrm{HCl}$.

\section{ESTIMATION OF ADOMET, ADOHCY AND ADENOSINE}

AdoMet, AdoHcy and adenosine content of different herbs and spices were quantitatively estimated by cation exchange High Performance Liquid Chromatography (HPLC) [22]. The HPLC column used was Partisil 10SCX, $4.6 \times 250 \mathrm{~mm}$, Whatman Inc., England, fitted to an HPLC system consisting of two pumps (Model 515), a Rheodyne injector and a programmer controlled by pump control module (PCM). Elution of AdoMet was monitored in-line by measurement of absorbance at 259 $\mathrm{nm}\left(\mathrm{A}_{259}\right)$, using a dual wavelength UV-Visible detector (Model 2487). Elution times as well as peak quantification were obtained from the Millenium ${ }^{32}$ software. All these HPLC equipments were from Waters, USA.

AdoMet, AdoHcy and adenosine were eluted near $31.617 \mathrm{~min}, 10.998 \mathrm{~min}$ and $8.993 \mathrm{~min}$. The amounts of standard compounds AdoMet, AdoHcy and adenosine (expressed in $\mu \mathrm{g}$ ) were plotted along the $\mathrm{x}$-axes and respective peak areas were plotted along the $y$-axes and compared with the samples (in triplicate) from different plant extracts. The standard solutions of AdoMet (Sigma, USA), AdoHcy (Sigma, USA) and adenosine (Sigma, USA) were prepared by dissolving in $0.01 \mathrm{~N} \mathrm{HCl}$.

Different volumes $(5,10,20 \mu \mathrm{l})$ of the standard compounds of known concentrations $(0.435 \mathrm{mM}$ AdoMet, $2.17 \mathrm{mM}$ AdoHcy and $0.9 \mathrm{mM}$ adenosine) were injected into the HPLC system to get standard curves. Values obtained for test sample (in triplicate) were derived from the standard curve when $r^{2}$ (square of correlation coefficient) values were near 0.99 .

The amount of trehalose present in the samples was estimated using acid trehalase (AT) enzyme, purified in our laboratory according to published method [23,24]. The standard aqueous trehalose (Sigma USA) solution $(1 \mathrm{mg} / \mathrm{ml}$ ) was prepared. Values represented here are average of experiments done in triplicate.

Oleanolic acid was estimated by Thin Layer Chromatographic (TLC) method [25] with some modifications. Different volumes $(2.5,5.0,10.0 \mu \mathrm{l})$ of standard oleanolic acid $(0.9 \mathrm{mg} / \mathrm{ml}$ chloroform; Sigma, USA) were spotted on a precoated alumina TLC plate along with different plant extracts ( $1 \mathrm{~g}$ dried powder/ml chloroform), $10 \mu \mathrm{l}$ were spotted on different lanes. The optimized solvent system for best separation of oleanolic acid from the other chemical constituents present in chloroform extract of the different plants was the combination of benzene, chloroform and ethyl acetate in the ratio of $6: 3: 1$. After complete air-drying of the TLC plate, it was sprayed by the anisaldehyde-sulfuric acid reagent for derivatization and the plate was immediately taken to a heating chamber $\left(100^{\circ} \mathrm{C}\right)$ for $5.0 \mathrm{~min}$ and scanned in a HP scanjet (Model \# 4570c) scanner at a resolution of $600 \mathrm{dpi}$ and densitometrically quantified by a NIH (National Institute of Health, USA) make software, namely "ImageJ". Different amounts of oleanolic acid were plotted along the $\mathrm{x}$-axis and the respective total pixel counts (pixel density $\mathrm{x}$ area of the spot $\left(\mathrm{R}_{\mathrm{f}}=0.22\right)$ ) were plotted along the $y$-axis to get a standard curve. Putting the values of total pixel counts on the standard curve the amounts of oleanolic acid present in different plant parts were estimated. The identification of oleanolic acid was done by mass spectrometry after scraping the spot in the TLC plate co-linear with the spots of standard oleanolic acid using ESI-MS analysis in a LC-QTOF system, Micromass, UK and ${ }^{1} \mathrm{H}$ NMR (300 MHz) study was performed on a DPX 300 NMR instrument, Bruker, Germany using tetra methyl silane (TMS; Sigma, USA) as internal standard.

\section{RESULTS AND DISCUSSION}

Among the herbs and spices studied, C. roseus leaves contained highest quantity of AdoMet $(1.63 \mathrm{mg} / \mathrm{g})$ (Ta- 
ble 1). Moderate to low AdoMet has been found in the descending order of $O$. sanctum $>A$. sativum $>T$. cordifolia $>W$. somnifera $>A$. cepa $>$ P. kurroa $>Z$. officinale $>E$. erecta. $C$. roseus was not only rich in AdoMet but this plant also had highest AdoHcy $(0.347 \mathrm{mg} / \mathrm{g}$ leaf $)$ and adenosine $(0.084 \mathrm{mg} / \mathrm{g}$ leaf $)$, the metabolic products of AdoMet. The rest of the plants had either low or moderate quantities of AdoHcy which decreased in the order of $W$. somnifera $>A$. sativum $>O$. sanctum $>A$. cepa $>A$. sativum $>E$. erecta $>T$. cordifolia $>Z$. officinale. No AdoHcy and adenosine were detected in P. kurroa and no adenosine was detected in E. erecta under the assay conditions. Incidentally, these plants had little AdoMet as well. Besides $C$. roseus the decreasing order of adenosine content was $W$. somnifera $>A$. sativum $>A$. cepa $>O$. sanctum $>T$. cordifolia $>Z$. officinale.

Among the nine plants both $A$. cepa and $W$. somnifera had relatively higher trehalose content $(101.94 \mathrm{mg} / \mathrm{g}$ bulb and $45.08 \mathrm{mg} / \mathrm{g}$ root respectively). Moderate to low amount of trehalose was present in the rest of the plants and decreased in the order of $P$. kurroa $>C$. roseus $>T$. cordifolia $>Z$. officinale $>$ A. sativum $>O$. sanctum $>E$. erecta.

O. sanctum appeared to be the only plant which contained oleanolic acid $\left(10.2 \mathrm{mg} / \mathrm{g}\right.$ leaf) (blue spot at $\mathrm{R}_{\mathrm{f}}=$ 0.22 ) among all the herbs and spices studied.

The leaf of $C$. roseus is well known for the storage of alkaloids like vinblastine and vincristine, which have anti-tumor activities [26]. Through this study another bioactive product of $C$. roseus leaf viz. AdoMet has been detected. Leaves of $C$. roseus contained nearly forty-six fold higher AdoMet in comparison to average AdoMet content of the rest of the plants. Genetically modified Kluyveromyces lactis mutants have been recognized as a good source of AdoMet [27]. Similar genetic manipulations could be performed to get mutant $C$. roseus plants rich in AdoMet. Although $W$. somnifera contained very little amount of AdoMet $(0.037 \mathrm{mg} / \mathrm{g}$ leaf $)$ but it had both higher AdoHcy $(0.148 \mathrm{mg} / \mathrm{g}$ root) and adenosine ( $0.0508 \mathrm{mg} / \mathrm{g}$ root) content and the AdoHcy: AdoMet and adenosine: AdoMet ratios were remarkably high compared to other plants. So, it could be said that in $W$. somnifera, although AdoMet was synthesized in appreciable quantities but was mostly utilized for its own metabolic purposes. Both $A$. cepa and $W$. somnifera had twenty fold and nine fold higher trehalose content respectively compared to average trehalose content of the rest of the seven plants and these two plants could be exploited as alternative sources of trehalose as their trehalose content is comparable with other well known trehalose rich sources like yeast (10-15\% wt/dry wt) [28], fungi (7-10\% wt/dry wt) [29,30], etc. Occurrence of trehalose was reported to form a glassy state in the cell, in which degradative reactions and intracellular ice formation could sufficiently be arrested during prolonged storage. In India, bulb of $A$. cepa is one of the daily used food ingredients. This is the first report of $A$. сеpa bulbs containing such a huge percentage of trehalose. Since it is an economically favorable crop and its production rate is also very high, more trehalose enriched genetically improved Allium cepa could be cultivated in near future.

\section{ACKNOWLEDGEMENTS}

The authors express their hearty thanks to Prof. S. Roy, Director, Indian Institute of Chemical Biology, Kolkata for providing infrastructural facilities. Authors thank D \& P program of Department of Science $\&$ Technology, Govt. of India and Dey's Medical Stores (Mfg.) Ltd.,

Table 1. Estimation of AdoMet, AdoHcy, Adenosine, Trehalose and Oleanolic acid.

\begin{tabular}{lccccc}
\hline Names of the Plants & $\begin{array}{c}\text { AdoMet } \mathbf{( \% ,} \\
\mathbf{w t} / \mathbf{w t}) \times \mathbf{1 0}^{-4}\end{array}$ & $\begin{array}{c}\text { AdoHcy } \\
(\mathbf{\%}, \mathbf{w t} / \mathbf{w t}) \\
\times \mathbf{1 0}^{-\mathbf{4}}\end{array}$ & $\begin{array}{c}\text { Adenosine } \\
(\mathbf{\%}, \mathbf{w t} / \mathbf{w t}) \\
\times \mathbf{1 0}\end{array}$ & $\begin{array}{c}\text { Trehalose } \\
(\mathbf{\%}, \mathbf{w t} / \mathbf{w t}) \\
\times \mathbf{1 0}\end{array}$ & $\begin{array}{c}\text { Oleanolic acid } \\
(\mathbf{\%}, \mathbf{w t} / \mathbf{w t})\end{array}$ \\
\hline Tinospora cordifolia & $51.37 \pm 0.21$ & $0.90 \pm 0.014$ & $5.50 \pm 0.0076$ & $59.10 \pm 0.06$ & n.d. \\
Picrorrhiza kurroa & $9.18 \pm 0.03$ & n.d. & n.d. & $99.0 \pm 0.06$ & n.d. \\
Eclipta erecta & $6.36 \pm 0.05$ & $1.03 \pm 0.006$ & n.d. & $1.01 \pm 0.01$ & n.d. \\
Ocimum sanctum & $79.68 \pm 0.30$ & $7.73 \pm 0.01$ & $6.83 \pm 0.01$ & $17.05 \pm 0.03$ & $1.02 \pm 0.02$ \\
Allium cepa & $21.52 \pm 0.17$ & $4.78 \pm 0.08$ & $7.08 \pm 0.02$ & $1019.43 \pm 0.13$ & n.d. \\
Zingiber officinale & $6.58 \pm 0.05$ & $0.36 \pm 0.01$ & $2.22 \pm 0.01$ & $59.03 \pm 0.06$ & n.d. \\
Allium sativum & $69.38 \pm 0.20$ & $1.76 \pm 0.01$ & $25.34 \pm 0.15$ & $38.11 \pm 0.03$ & n.d. \\
Withania somnifera & $37.29 \pm 0.23$ & $148.47 \pm 0.35$ & $50.82 \pm 0.12$ & $450.77 \pm 0.19$ & n.d. \\
Catharanthus roseus & $1630.42 \pm 0.23$ & $346.77 \pm 0.43$ & $448.29 \pm 0.19$ & $84.40 \pm 0.29$ & n.d. \\
\hline
\end{tabular}

n.d. - not detected 
Kolkata for their financial assistance. Thanks also given to Network Project 0005, Council of Scientific and Industrial Research, Govt. of India for providing financial assistance to $\mathrm{PC}$ and TD.

\section{REFERENCES}

[1] Lu, S.C. (2000) S-Adenosylmethionine. The International Journal of Biochemistry \& Cell Biology, 32(4), 391-395.

[2] Fernandez-Checa, J.C., Colell, A. and Garcia-Ruiz, C. (2002) S-Adenosyl-L-methionine and mitochondrial reduced glutathione depletion in alcoholic liver disease. Alcohol, 27(3), 179-183.

[3] Turchetti, V., Bellini, M.A., Leoncini, F., Petri, F., Trabalzini, L., Guerrini, M. and Forconi, S. (2000) Blood viscosity and red cell morphology in subjects suffering from cirrhosis before and treatment with S-adenosyl-Lmethionine (SAM). Clinical Hemorheology and Microcirculation, 22(3), 215-221.

[4] Mischoulon, D. and Fava, M. (2002) Role of S-adenosylL-methionine in the treatment of depression: A review of the evidence. American Journal of Clinical Nutrition, 76(5), 11585-11615.

[5] Fuso, A., Seminara, L., Cavallaro, R.A., D'Anselmi, F. and Scarpa, S. (2005) S-adenosylmethionine/homocysteine cycle alterations modify DNA methylation status with consequent deregulation of PS1 and BACE and beta-amyloid production. Molecular and Cellular Neuroscience, 28(1), 195-204.

[6] Najm, W.I., Reinsch, S., Hoehler, F., Tobis, J.S. and Harvey, P.W. (2004) S-adenosyl methionine (SAMe) versus celecoxib for the treatment of osteoarthritis symptoms: a double-blind cross-over trial. BMC Musculoskeletal Disorders, $\mathbf{5 ( 1 )}, 6$.

[7] Kaushik, J.K. and Bhat, R. (2003) Why Is trehalose an Exceptional Protein Stabilizer? An analysis of the thermal stability of proteins in the presence of the compatible osmolyte trehalose. Journal of Biological Chemistry, 278(29), 26458-26465.

[8] Morana, A., Stiuso, P., Colonna, G., Lamberti, M., Carten, M. and De Rosa, M. (2002) Stabilization of S-adenosylL-methionine promoted by trehalose. Biochimica et Biophysica Acta, 1573(2), 105-108.

[9] Limaye, L.S. and Kale, V.P. (2001) Cryopreservation of human hematopoietic cells with membrane stabilizers and bioantioxidants as additives in the conventional freezing medium. Journal of Hematotherapy and Stem Cell Research, 10(5), 709-718.

[10] Liu, J. (1995) Pharmacology of oleanolic acid and ursolic acid. Journal of Ethnopharmacology, 49(2), 57-68.

[11] Giner-Larza, E.M., Manez, S., Recio, M.C., Giner, R.M., Prieto, J.M., Cerda-Nicolas, M. and Rios, J.L. (2001) Oleanonic acid, a 3-oxotriterpene from Pistacia, inhibits leukotriene synthesis and has anti-inflammatory activity. European Journal of Pharmacology, 428(1), 137-143.

[12] Hsu, H.Y., Yang, J.J. and Lin, C.C. (1997) Effects of oleanolic acid and ursolic acid on inhibiting tumor growth and enhancing the recovery of hematopoietic system postirradiation in mice. Cancer Letter, 111(1-2), $7-13$.
[13] Ubaid, R.S., Anantrao, K.M., Jaju, J.B. and Mateenuddin, M. (2003) Effect of Ocimum sanctum (OS) leaf extract on hepatotoxicity induced by antitubercular drugs in rats. Indian. Journal of Physiology and Pharmacology, 47(4), 465-470.

[14] Singh, S.S., Pandey, S.C., Srivastava, S., Gupta, V.S., Patro, B. and Ghosh, A.C. (2003) Chemistry and medicinal properties of Tinospora cordifolia (guduchi). Indian Journal of Pharmacology, 35(2), 83-91.

[15] Thyagarajan, S., Jayaram, S., Gopalakrishnan, V., Hari, R., Jeyakumar, P. and Sripathi, M. (2002) Herbal medicines for liver diseases in India. Journal of Gastroenterology and Hepatology, 17(S3), S370- S376.

[16] Akbarsha, M.A., Vijendrakumar, S., Kadalmani, B., Girija, R. and Faridha, A. (2000) Curative property of Withania somnifera Dunal root in the context of carbendazim-induced histopathological changes in the liver and kidney of rat. Phytomedicine, 7(6), 499-507.

[17] Bhattacharyya, D., Mukherjee, R., Pandit, S., Das, N. and Sur, T.K. (2003) Prevention of carbon tetrachloride induced hepatotoxicity in rats by himoliv, a polyherbal formulation. Indian Journal of Pharmacology, 35(3), 183-185.

[18] Balasenthil, S. and Nagini, S. (2000) Garlic exerts hepatoprotective effects during 4-nitroquinoline 1-oxideinduced oral carcinogenesis in rats. Asia Pacific Journal of Clinical Nutrition, 9(2), 136-138.

[19] Al-Yahya, M.A., Rafatullah, S., Mossa, J.S., Ageel, A.M., Parmar, N.S. and Tariq, M. (1989) Gastroprotective activity of ginger zingiber officinale rosc in albino rats. American Journal of Chinese Medicine, 17(1-2), 51-66.

[20] Zheng, W.Z. and Wang, S.Y. (2001) Antioxidant Activity and Phenolic Compounds in Selected Herbs. Journal of Agricultural and Food Chemistry, 49(11), 5165-5170.

[21] Sutherland, I.W. and Wilkinson, J.F. (1971) Chemical extraction methods of microbial cells. Methods in Microbiology, 5(Part 2), 345-383.

[22] Hoffman, J.L. (1986) Chromatographic analysis of the chiral and covalent instability of S-adenosyl-L-methionine. Biochemistry, 25(15), 4444-4449.

[23] Bhattacharyya, S., Chaudhuri, P., Basu, A. and Ghosh, A.K. (2005) Regulation of trehalose metabolism by Adox and AdoMet in Saccharomyces cerevisiae. Indian Journal of Experimental Biology, 43(4), 360-368.

[24] Biswas, N. and Ghosh, A.K. (1996) Characterisation of an acid trehalase of Saccharomyces cerevisiae present in trehalase-sucrase aggregate. Biochimica et Biophysica Acta, 1290(1), 95-100.

[25] Baricevic, D., Sosa, S., Loggia, R.D., Tubaro, A., Simonovska, B., Krasna, A. and Zupancic (2001) A Topical anti-inflammatory activity of Salvia officinalis L. leaves: The relevance of ursolic acid. Journal of Ethnopharmacology, 75(2), 125-132.

[26] Dutta, A., Batra, J., Pandey-Rai, S., Singh, D., Kumar, S. and Sen, J. (2005) Expression of terpenoid indole alkaloid biosynthetic pathway genes corresponds to accumulation of related alkaloids in Catharanthus roseus (L.) G. Don. Planta, 220(3), 376-383.

[27] Mincheva, K.P. and Balutsov, V.M. (2002) Isolation of a Kluyveromyces lactis Mutant Enriched in S-Adenosyl-LMethionine and Growing in Whey Medium. Applied 
Biochemistry and Microbiology, 38(4), 335-338.

[28] Savova, I., Donev, T. and Sholeva, Z. (1998) An investigation of the trehalose accumulation dynamics by yeasts from genus Saccharomyces. Journal of Culture Collections, 2(1), 40-43.

[29] Clegg, J.S. and Filosa, M.F. (1961) Trehalose in the cel- lular slime mold, Dictysostelium mucoroides. Nature, 192(4807), 1077-1078.

[30] Sussman, A.S. and Lingappa, B.T. (1959) Role of trehalose in ascospores of Neurospora tetrasperma. Science, 130(3385), 1343-1344. 